\title{
Determinación de los niveles de riesgo socioecológico ante sequías en sistemas agrícolas campesinos de La Araucanía chilena. Influencia de la diversidad cultural y la agrobiodiversidad
}

\author{
René Montalba \\ Universidad de La Frontera. Departamento de Ciencias Agronómicas y Recursos Naturales. \\ Núcleo Científico Tecnológico en Ciencias Sociales \\ rene.montalba@ufrontera.cl (autor de contacto) \\ Francisca Fonseca \\ Universidad de La Frontera. Departamento de Ciencias Sociales \\ francisca.fonseca@ufrontera.cl \\ Marcia García \\ Universidad de la Frontera. Magister en Gestión de Recursos Naturales \\ marcia.garcia@ufrontera.cl

\section{Lorena Vieli} \\ Universidad de La Frontera. Departamento de Ciencias Agronómicas y Recursos Naturales \\ lorena.vieli@ufrontera.cl

\section{Miguel Altieri} \\ University of California. College of Natural Resources \\ agroeco3@berkeley.edu
}

\section{Resumen}

Este trabajo pretende fomentar el entendimiento de la interrelación entre riesgos ambientales generados por el cambio climático y la capacidad de utilizar unidades y sistemas agrícolas (como sistemas socioecológicos) para resistir a estas perturbaciones y persistir en el tiempo. Fueron evaluadas 177 familias que practicaban agricultura campesina de la región de La Araucanía (Chile) y que estaban diferenciadas, de acuerdo con su origen étnico, en mapuches, chilenos y descendientes de colonos europeos. Se identificaron y se evaluaron variables asociadas a los niveles de ocurrencia y de intensidad de sequías (amenaza) y la vulnerabilidad de los sistemas campesinos ante estos eventos, así como su capacidad de respuesta. Las técnicas de recolección de información incluyeron información secundaria, SIG, encuesta, observación directa, entrevista individual y entrevista 
grupal. Los valores obtenidos fueron integrados algorítmicamente y se generó un índice de riesgo socioecológico (IRSE), con valores desde 0 hasta 2 . Un $48 \%$ de los predios presentó valores de IRSE bajos (0-0,7), mientras que los restantes se encontraron en niveles medios (0,7-1,3), lo cual puede ser vinculado a altos grados de resiliencia. En relación con las variables de amenaza, vulnerabilidad y capacidad de respuesta, se encontraron diferencias estadísticamente significativas (ANDEVA) entre grupos de campesinos, lo cual está relacionado principalmente con el origen étnico de estos. En general, los campesinos mapuches presentaron menor vulnerabilidad y mayores valores de capacidad de respuesta que los chilenos y los colonos, lo cual se relaciona con el mayor nivel de conocimiento de prácticas específicas y la mantención en sus sistemas productivos de especies o variedades tolerantes a la escasez hídrica.

Palabras clave: resiliencia; biodiversidad; agricultura campesina; multiculturalismo; gestión del riesgo.

Abstract. Determining levels of drought-related socio-ecological risk of Araucania farming systems of Chile: Influence of cultural diversity and agro-biodiversity

This research attempts to increase our understanding of the relationship between environmental risks caused by climate change and the capacity of agricultural systems to resist these disturbances and persist in time. A total of 177 agricultural units were evaluated in the Araucania Region of Chile. The ethnical origin of the owners of these units was classified as Mapuche, Chilean or descendants of European colonizers. Variables associated with the level of occurrence and intensity of droughts (threat) and the vulnerability of the agricultural systems to these events were also assessed, as well as their response capacity. This information was produced using secondary information, GIS, direct measurement, surveys, and individual and group interviews. The values of the variables measured were integrated to develop a "Socio-Ecological Risk Index" (IRSE) with values that ranged from 0 to 2 . When calculating this index for each farm evaluated, $48 \%$ presented low values (0-0.7), while the rest showed intermediate values (0.7-1.3). The intermediate values may be related to higher levels of resilience. Variables related to threat, vulnerability and response capacity showed significant differences (ANDEVA) between the ethnic groups. In general, Mapuche farmers presented lower vulnerability values and higher response capacity values than Chilean and descendants of European colonizers, which was mainly related to the Mapuche's knowledge of specific management techniques and their tendency to conserve species or varieties of crops tolerant to water scarcity.

Keywords: resilience; biodiversity; small farms; multiculturalism; risk management.

\section{Sumario}
1. Introducción
4. Conclusiones
2. Metodología
Agradecimientos
Referencias bibliográficas

3. Resultados y discusión 


\section{Introducción}

Uno de los temas de mayor interés social, ciudadano, político, económico y científico corresponde al estudio de impactos ambientales que derivan del modelo de crecimiento dominante, basado en un uso intensivo de recursos naturales y materias primas. El conocimiento científico y la información que se obtiene de este nos evidencian que los impactos negativos en el medio ambiente son cada vez mayores en cantidad e intensidad, y su escala ya abarca a todo el planeta. De esta forma, en la actualidad, se está prestando mucha atención a las capacidades o a los potenciales que tendrían distintos tipos de sistemas socioecológicos para absorber perturbaciones y cambios que les afectaran y de persistir sin variaciones cualitativas en su estructura (Young et al., 2006; Holling, 1973), así como también de generar una «memoria social» (en la forma de prácticas o estructuras sociales de apoyo) para hacer frente a perturbaciones futuras similares (Hammond et al., 2013). Este atributo de los sistemas socioecológicos ha sido denominado «resiliencia socioecológica» (Folke, 2006).

\section{Riesgos ecológicos y modernidad reflexiva}

Para autores como Beck, Luhmann, Giddens, Bauman o Lash, la característica definitoria de la modernidad reflexiva es la ocurrencia de riesgos fabricados por la propia civilización humana. Estos riesgos son efectos colaterales de los procesos industriales y han llegado a poner en peligro los requisitos básicos para la vida en general y para la vida social en particular (Beck, 2006; Giddens et al., 1996; Bauman, 2006). Con ello, la «naturaleza» ha dejado de ser un opuesto situado fuera del orden social, para tomar la forma de diversos y complejos problemas ambientales que provienen desde el interior de la propia sociedad.

Los problemas del medio ambiente [ya] no son problemas del entorno, sino [en su génesis y consecuencias] problemas sociales, problemas del ser humano, de su historia, de sus condiciones de vida, de su referencia al mundo y a la realidad, de su ordenamiento económico, cultural y político. (Beck, 2006)

La teoría de la sociedad del riesgo concibe el fenómeno del riesgo como algo global. Peligros globales - como el nuclear, el químico, el genético y el ecológico- son tanto imprevisibles como inevitables, lo que resquebraja los pilares del sistema de seguridad. Por ello, los daños no solo afectan a las sociedades de modernización avanzada, sino también a las de modernización periférica, pues ambas carecen de dispositivos para prevenir y controlar los riesgos ecológicos que autoproducen.

La generación de mecanismos y de estrategias de control y prevención del riesgo ecológico se ha transformado en una preocupación importante para las instituciones internacionales y los gobiernos nacionales, así como para los individuos y las comunidades de individuos que generan o recuperan prácticas más ecológicas para enfrentar los riesgos y la incertidumbre que estos producen. 


\section{Riesgo global y agricultura «moderna»}

En los países industrializados, la agricultura ha sido fuertemente protegida de los efectos de la liberalización (Josling y Tangermann, 1999). El enfoque neoclásico de desarrollo agrícola, implantado en los últimos tiempos (desde los años 50), ha alentado a la retirada de la intervención del Estado respecto de la gobernanza del mercado y de los sistemas de producción (Friedmann, 1998). Con el objetivo de mejorar la eficiencia y maximizar la rentabilidad económica de la agricultura, el llamado modelo liberal-productivista propende a la liberalización del mercado y a una mayor racionalización de los métodos de producción y comercio. Desde esta perspectiva, la diversidad natural (biológica, cultural y ambiental) es considerada una parte de las ineficiencias a superar, lo cual se realizaría mediante la «modernización» agrícola y el desarrollo de sistemas de agricultura industrial, con alta utilización de insumos (semillas, agroquímicos y energía), mecanización y control de procesos (Guzmán et al., 2000).

Existe preocupación respecto a la globalización de la agricultura dentro del enfoque neoclásico, dado que se estaría consolidando la unificación y la homogeneización de las prácticas agrícolas y los agroecosistemas, a través de la introducción de nuevas técnicas y prácticas comerciales que se aplican a nivel mundial y en función de las demandas de las economías de escala (Schnaiberg y Gould, 1994; Athanasiou, 1996). La globalización de la agricultura se inserta dentro de las crisis ambiental y social, las cuales van asociadas, principalmente, con la incapacidad del sistema moderno de considerar y valorar la diversidad agroecológica. Así, el desarrollo del mercado global afectaría mayormente a aquellos que no tienen los recursos para mejorar su capacidad de producción dentro de límites ecológicamente sostenibles. El éxito de la aplicación del paradigma neoclásico se enfocaría, una vez más, en los agricultores con más recursos, en las comunidades más ricas, en las zonas más aptas para la agricultura moderna, de las sociedades con mayor crecimiento y capaces de incorporar prácticas esenciales para el desarrollo eficaz y sostenible (Guzmán et al., 2000). En un mercado global y con fuerzas del mercado no regulado, muchos sistemas agrícolas poseen pocas probabilidades de sobrevivir, debido a que los sistemas más eficientes para esas condiciones gozarán de ventajas competitivas, tales como una mayor abundancia de recursos naturales, el apoyo industrial eficaz o menores costos de producción (Ramakrishnan, 1999).

Los posibles riesgos de este escenario se ven agravados por la evolución de las bases científicas y tecnológicas de la agricultura, las cuales están cada vez más concentradas en unas pocas empresas multinacionales. De esta forma, la seguridad agroalimentaria está quedando en manos de empresas cuyo objetivo principal no es la prestación de bienes y servicios a las poblaciones locales, sino más bien la extracción de ganancias (Brenner, 1997; Paillotin, 1998; Altieri, 1999; Caswell, 2000). Esta temática debe, entonces, ser planteada y considerada como parte del contexto de las nuevas estructuras agroalimentarias que se plantean para el siglo XXI, considerando las fallas sistémicas del modelo de desarrollo dominante. En este contexto, resulta necesaria la consideración, el estudio y la conservación de 
la diversidad biológica y de la agrobiodiversidad local, además de la diversidad cultural, ya que estas resultan elementos indispensables que deben estar disponibles para el restablecimiento y la recuperación de procesos y sistemas resilientes y sustentables de producción de alimentos (Altieri et al., 2014).

\section{Agricultura tradicional campesina, modernización, riesgo y resiliencia socioecológica}

Bajo el concepto de "agricultura tradicional» (o "precientífica»), podemos agrupar a aquellos tipos de agricultura o sistemas agrícolas que, tanto en su desarrollo como en su funcionamiento, no responden a la lógica científica ni económico-mercantilista de los sistemas «modernos», sino que más bien obedecen a concepciones de hombre y de naturaleza diversos, que se han originado y se han desarrollado a través del tiempo mediante múltiples relaciones entre los diferentes grupos y el medio que habitan (Altieri, 1999). De este modo, en la agricultura tradicional, el desarrollo de sus sistemas es el resultado de una coevolución —en el sentido de evolución integrada - entre cultura y medio ambiente (Sevilla Guzmán y González de Molina, 1990). Esta se ha beneficiado de siglos de evolución cultural y biológica, a través de la cual se ha adaptado a las condiciones locales. Así, los agricultores han creado y/o heredado sistemas complejos de agricultura que, durante siglos, los han ayudado a satisfacer sus necesidades de subsistencia, incluso bajo condiciones ambientales muy adversas (Altieri, 1999). Este proceso ha contribuido a originar diversas culturas en interacción con medios ambientes también diversos, en los cuales, y por medio de la estrecha relación entre los agricultores y la naturaleza, los humanos han desarrollado un vasto conocimiento de las distintas especies naturales y sus ecosistemas, domesticando una gran diversidad de especies y variedades de estas (Altieri et al., 2014).

$\mathrm{La}$ «agricultura moderna» se mundializa a partir de la década de 1960 por medio de la denominada «revolución verde», ya que, por medio de esta, logran ser transferidos a la agricultura los patrones y los modelos productivos de la industria: $a$ ) economía de escala; $b$ ) modelos y sistemas productivos genéricos homogéneos en base a insumos petroquímicos, y c) maximización de la productividad e ingresos crematísticos (Guzmán et al., 2000). Dentro de los efectos colaterales de este proceso, se encuentra el desplazamiento de agricultores campesinos. No obstante, el desplazamiento de unidades de agricultura tradicional (campesina) ha sido mucho más intenso en zonas más adecuadas para los «sistemas productivos modernos» (suelos planos, fértiles, con disponibilidad de agua y alto potencial productivo), y las unidades de menor escala y los sistemas tradicionales han quedado en sitios «marginales» que presentan limitaciones productivas y fragilidad ecológica: pendientes altas a medias; suelos delgados, poco productivos y frágiles; baja retención o escasez de recursos hídricos; temperaturas muy bajas o muy altas; etc. (Altieri, 1999; Montalba, 2011). De esta forma, es posible considerar que los sistemas agrícolas campesinos o de agricultura tradicional habrían desarrollado prácticas culturales y 
productivas, así como también formas de organización o estructuras sociales que los han ayudado a hacer frente a estas limitaciones. Del mismo modo, se puede plantear como hipótesis que estas adaptaciones y estructuras de organización pueden ser relevantes al momento de amortiguar, resistir o recobrarse de los efectos locales de cambios globales, como la variación en los regímenes térmicos o la reducción de recursos hídricos.

Desconociendo este potencial de los sistemas de agricultura tradicional, por lo general, desde los organismos oficiales o de gobierno, se considera que los sistemas agrícolas menos tecnificados resultan ser mucho más susceptibles y menos adaptables al cambio climático que los modernos e intensivos (Vargas, 2007; Ministerio Nacional de Agricultura, 2010b). Hipótesis alternativas consideran que, debido a la adaptación a las condiciones de marginalidad, precariedad y cambio continuo, los sistemas campesinos habrían desarrollado prácticas culturales, estrategias adaptativas y formas de organización socioproductiva que les conferirían mayor tolerancia, capacidad de recuperación y adaptación a los cambios ambientales que la que presentan los sistemas modernos de agricultura (Nicholls y Altieri, 2013). Estudios sobre los impactos del cambio climático en la agricultura (Altieri y Nicholls, 2009; Nicholls et al., 2012) han encontrado que agricultores "campesinos» de distintas áreas geográficas desarrollarían estrategias de adaptación a la escasez hídrica, lo cual minimizaría las pérdidas en productividad mediante el desarrollo de prácticas culturales como la conservación de variedades locales tolerantes a la sequía, asociaciones de cultivos, sistemas de acumulación y redistribución de agua y una serie de otras prácticas culturales y organizacionales. Ante estos resultados, se considera necesario identificar, sistematizar y evaluar los impactos de estas prácticas campesinas sobre la capacidad adaptativa que manejan los campesinos (indígenas y no indígenas) para enfrentar los cambios ambientales (Altieri y Nicholls, 2009) y permanecer en el tiempo.

\section{La Araucanía chilena como laboratorio en vivo}

La región de La Araucanía chilena se ubica a $700 \mathrm{~km}$ al sur de Santiago y presenta una superficie cercana a los 5 millones de hectáreas. En este territorio, se conjugan elementos bioecológicos, históricos y socioculturales que la convierten en una zona de una importancia particular para entender la relación entre modernización, cambios globales y sus efectos en sistemas familiares campesinos, así como también las respuestas adaptativas generadas por estos:

a) Se inserta en una de las zonas consideradas por la UNESCO como centro de interés en biodiversidad.

b) Tiene una alta variabilidad de condiciones agroecológicas necesarias para el desarrollo de los cultivos.

c) Posee registros tempranos (1550) de incorporación de cultivos alimenticios (trigo, cebada, manzanos, etc.) y de una alta adopción cultural por parte de grupos locales. 
d) Muestra una diversidad cultural única en Chile, representada por pueblos originarios (mapuches y pewenches), descendientes de colonos extranjeros (alemanes, suizos, italianos, franceses) y chilenos, establecidos en la región, principalmente, en el último siglo.

e) Presenta una alta diversidad en tipos de explotación y orientaciones agrícolas (comuneros, campesinos, agricultura tradicional cerealera, agricultura de exportación y grandes empresas agrícolas y forestales).

f) En el último siglo, se han desarrollado una serie de procesos vinculados a la modernización y a la globalización productiva (reducción indígena, cerealización y expansión forestal) (Montalba y Stephens, 2014).

Asociados a estos procesos y a los efectos del cambio climático global, la escasez hídrica para la producción agrícola y el consumo humano ha sido destacada por las autoridades locales como uno de los principales problemas de la región (Montalba et al., 2015). De esta forma, en el presente trabajo, se plantea la utilización de La Araucanía como un laboratorio en vivo para estudiar los procesos de adaptación y los niveles comparados de resiliencia socioecológica de los distintos tipos de sistemas agrícolas tradicionales campesinos.

\section{Metodología}

\section{Caracterización bioclimática del área de estudio}

El área de estudio comprende dos zonas de la región de La Araucanía (Chile), ubicadas entre los $37^{\circ} 35^{\prime}$ y los $39^{\circ} 35^{\prime}$ de latitud sur y desde $70^{\circ} 50^{\prime}$ de longitud oeste, las cuales presentan características climáticas contrastantes. Por una parte, la comuna de Lumaco se ubica en el denominado «secano interior» y destaca por presentar una precipitación de 800 a $900 \mathrm{~mm}$ anuales y una estación seca prolongada, que se extiende de 5 a 6 meses o más a partir de octubre, $y$, por otra parte, la comuna de Curarrehueque se ubica en la zona agroecológica denominada "cordillera de los Andes», la cual presenta precipitaciones cercanas a los $2.000 \mathrm{~mm}$ anuales y solo de 2 a 3 meses de déficit hídrico (Rouanet et al., 1988) (figura 1).

\section{Evaluación de riesgo socioecológico en sistemas campesinos}

Para evaluar el riesgo socioecológico a nivel predial (RSE), el estudio se basó en el enfoque de Altieri (2013), el cual depende de tres variables: amenaza, vulnerabilidad y capacidad de respuesta. La amenaza representa la probabilidad de que ocurra un evento (intensidad, frecuencia), frente al cual esa comunidad en particular y sus predios son vulnerables. La vulnerabilidad representa la intensidad de los daños que puede producir la ocurrencia efectiva del proceso. La capacidad de respuesta representa los atributos que posee un predio y las estrategias y los manejos que usan los productores para reducir los riesgos de eventos climáticos, así como para resistir y recuperarse de los daños causados por dichos eventos; también puede considerarse la capacidad de adaptación de un sistema predial. 
Figura 1. Plano de ubicación de las áreas de estudio y de las unidades evaluadas

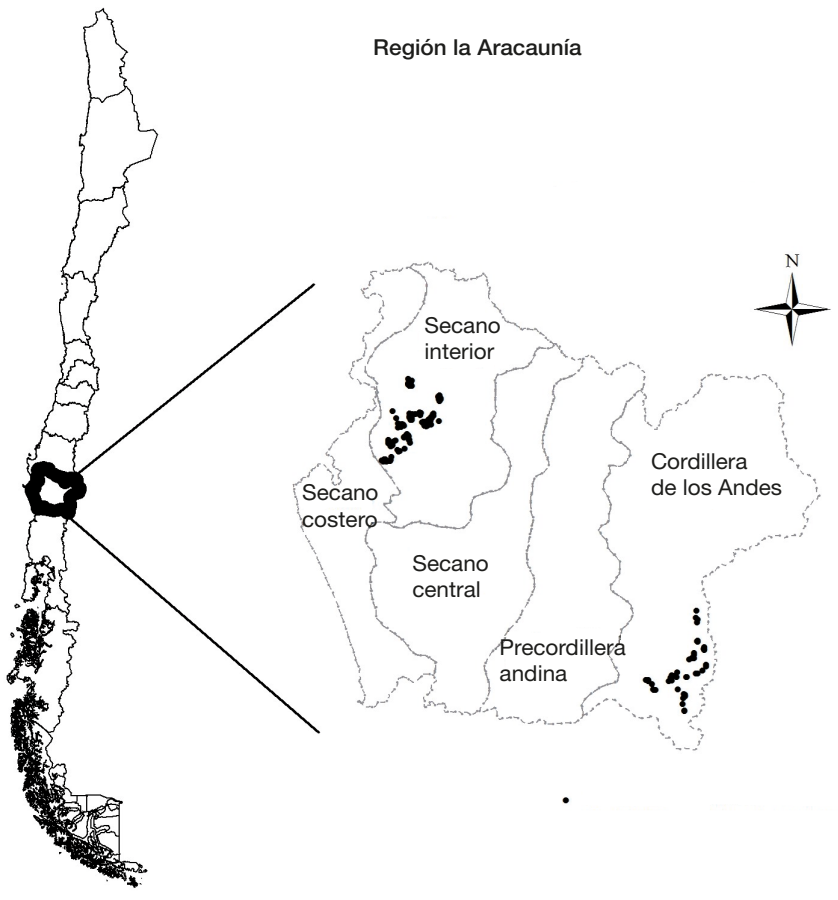

Cada uno de los componentes asociados a este riesgo socioecológico se midió en base a variables identificadas y seleccionadas en consulta y de acuerdo con campesinos y organizaciones de agricultores presentes en el territorio. En base a su naturaleza, estas variables fueron evaluadas utilizando información cartográfica, observación y medición directa en terreno, cuestionario socioeconómico, aplicación de ficha de manejo agronómico y entrevista individual o grupal e información secundaria proveniente de fuentes oficiales. De esta forma, las variables para evaluar la amenaza fueron:

a) Intensidad de la sequía (duración en meses).

b) Frecuencia de sequías (número de eventos por año).

c) Reducción de rendimiento de los cultivos (calculada en tantos por ciento).

Todas se determinaron por medio de la encuesta socioproductiva y la percepción de los campesinos respecto de la tendencia mayoritaria en los últimos 10 años y la corroboración por medio de estadísticas oficiales.

Las variables utilizadas para medir la vulnerabilidad correspondieron a:

a) Superficie de plantaciones forestales (porcentaje de superficie en un radio de $1 \mathrm{~km}$ ), utilizando la cobertura de plantaciones forestales (Conaf-Conama, 
2012). Con la ubicación del predio encuestado, se determinó el porcentaje del área ocupado por plantaciones forestales en un radio de $1 \mathrm{~km}$. Cabe destacar que, en la zona de estudio, se ha indicado a las plantaciones forestales como un importante factor de reducción de los recursos hídricos.

b) Acceso al agua. Fue determinado por medio de un formulario socioproductivo y la percepción de los campesinos respecto de su tendencia en los últimos 10 años (entrevistas).

c) Contexto agroecológico. Fue determinado por la evaluación a nivel predial de tres elementos en terreno:

- Número de subsistemas productivos.

- Número de especies vegetales por cada uno de ellos.

- Número de variedades (o ecotipos) de cada especie cultivada y silvestre presente en cada subsistema.

Por último, la capacidad de respuesta se evaluó a través de:

a) Nivel de recuperación del sistema. Se relacionó con la identificación y la utilización de especies vegetales cultivadas y/o variedades (ecotipos locales) que resistan o se recuperen de períodos de sequía, obtenidos por medio del formulario socioproductivo, entrevistas y evaluación predial en el terreno.

b) Nivel de conocimiento y toma de medidas preventivas. Se relacionó con el conocimiento que tiene el agricultor y las medidas preventivas que este realiza en su predio para enfrentar las sequías. Se obtuvo por medio del formulario socioproductivo y entrevistas a campesinos.

c) Redes de apoyo asociadas. Se refiere a averiguar si el agricultor cuenta con redes sociales, organizaciones o instituciones (ONG, gobierno u otras) que lo apoyen en períodos de escasez hídrica o sequías. Estas variables también fueron obtenidas por medio del cuestionario socioproductivo y las entrevistas. Todas las variables se midieron en una escala entre 0 y 100 (tabla 1).

\section{Campaña de terreno e "identificación cultural» de las unidades agrícolas (predios)}

La campaña de terreno se realizó entre los meses de febrero de 2013 y marzo de 2014. Cada una de las unidades agrícolas (predios) fueron georreferenciadas para su posterior evaluación en relación con variables cartográficas. De acuerdo con la aplicación de técnicas de cálculo muestral representativo, fueron evaluados un total de 177 predios, 120 de los cuales correspondieron a la comuna de Lumaco y 57, a Curarrehue. El análisis fue realizado mediante una estrategia plurimetodológica en base a la aplicación de las técnicas de investigación mencionadas en la tabla 1. De acuerdo con criterios como su origen étnicocultural, su lenguaje y su religiosidad, la identificación de prácticas culinarias o la autoidentificación de los agricultores (determinado por medio de formulario socioproductivo y entrevistas), los predios fueron identificados como manejados por campesinos mapuches, chilenos o colonos europeos. 
Tabla 1. Variables utilizadas en el análisis de riesgo socioecológico, técnicas de evaluación y valores asignados a cada variable

\begin{tabular}{|c|c|c|c|c|c|}
\hline & Variable & Método y fuente & Descripción & Descripción del indicador & Valor \\
\hline \multirow{10}{*}{ 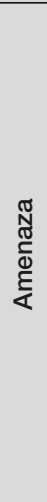 } & \multirow{4}{*}{$\begin{array}{l}\text { Intensidad } \\
\text { de la sequía }\end{array}$} & \multirow{4}{*}{$\begin{array}{l}\text { Formulario sociopro- } \\
\text { ductivo, entrevista, } \\
\text { estadísticas y } \\
\text { fuentes secundarias. }\end{array}$} & \multirow{4}{*}{$\begin{array}{l}\text { Considera la duración } \\
\text { del evento de déficit } \\
\text { hídrico. }\end{array}$} & Sin déficit hídrico. & 0 \\
\hline & & & & Déficit estival. & 33 \\
\hline & & & & Déficit en primavera y verano. & 66 \\
\hline & & & & Déficit permanente. & 100 \\
\hline & \multirow[t]{3}{*}{ Frecuencia } & \multirow{3}{*}{$\begin{array}{l}\text { Formulario sociopro- } \\
\text { ductivo, entrevista, } \\
\text { estadísticas y } \\
\text { fuentes secundarias. }\end{array}$} & \multirow{3}{*}{$\begin{array}{l}\text { Considera el número de } \\
\text { eventos por años (10 } \\
\text { años). }\end{array}$} & $\begin{array}{l}\text { Aumento de periodos } \\
\text { de escasez. }\end{array}$ & 100 \\
\hline & & & & $\begin{array}{l}\text { Mantención de periodos } \\
\text { de escasez. }\end{array}$ & 50 \\
\hline & & & & $\begin{array}{l}\text { Disminución de periodos } \\
\text { de escasez. }\end{array}$ & 0 \\
\hline & \multirow{3}{*}{$\begin{array}{l}\text { Pérdida de } \\
\text { rendimientos }\end{array}$} & \multirow{3}{*}{$\begin{array}{l}\text { Formulario sociopro- } \\
\text { ductivo y entrevista. }\end{array}$} & \multirow{3}{*}{$\begin{array}{l}\text { Considera el rendimien- } \\
\text { to de los } \\
\text { cultivos (quintales } \\
\text { por hectárea). }\end{array}$} & Aumento del rendimiento. & 0 \\
\hline & & & & Mantención del rendimiento. & 50 \\
\hline & & & & Disminución del rendimiento. & 100 \\
\hline \multirow{12}{*}{ 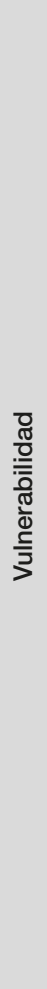 } & $\begin{array}{l}\text { Presencia de } \\
\text { plantaciones }\end{array}$ & $\begin{array}{l}\text { Cartografía (Conaf- } \\
\text { Conama, 2012). }\end{array}$ & $\begin{array}{l}\text { Considera las plantacio- } \\
\text { nes forestales en } \\
\text { un radio de } 1 \mathrm{~km} \text {. }\end{array}$ & $\begin{array}{l}\text { Porcentaje de superficie de } \\
\text { plantaciones circundantes al } \\
\text { predio. }\end{array}$ & $0-100$ \\
\hline & \multirow{4}{*}{$\begin{array}{l}\text { Acceso al } \\
\text { agua }\end{array}$} & \multirow{4}{*}{$\begin{array}{l}\text { Formulario sociopro- } \\
\text { ductivo y entrevista. }\end{array}$} & \multirow{4}{*}{$\begin{array}{l}\text { Considera suficiencia } \\
\text { de acceso de agua para } \\
\text { riego. }\end{array}$} & Muy bajo. & 100 \\
\hline & & & & Bajo. & 66 \\
\hline & & & & Medio. & 33 \\
\hline & & & & Alto. & 0 \\
\hline & \multirow{4}{*}{$\begin{array}{l}\text { Ubicación } \\
\text { del predio }\end{array}$} & \multirow{4}{*}{$\begin{array}{l}\text { Cartografía (Conaf- } \\
\text { Conama, 2012). }\end{array}$} & \multirow{4}{*}{$\begin{array}{l}\text { Considera la ubica- } \\
\text { ción del predio res- } \\
\text { pecto a la cuenca. }\end{array}$} & Cuenca alta. & 100 \\
\hline & & & & Ladera. & 66 \\
\hline & & & & Valle. & 33 \\
\hline & & & & Vega. & 0 \\
\hline & \multirow[t]{3}{*}{$\begin{array}{l}\text { Contexto } \\
\text { agroecológico }\end{array}$} & \multirow[t]{3}{*}{$\begin{array}{l}\text { Formulario sociopro- } \\
\text { ductivo, entrevista y } \\
\text { evaluación in situ. }\end{array}$} & \multirow{3}{*}{$\begin{array}{l}\text { Considera la evaluación } \\
\text { a nivel predial } \\
\text { de tres elementos } \\
\text { en el terreno: } \\
\text { 1. Número de } \\
\text { subsistemas } \\
\text { productivos. } \\
\text { 2. Número de } \\
\text { especies vegetales } \\
\text { por cada uno de } \\
\text { ellos. } \\
\text { 3. Número de } \\
\text { variedades } \\
\text { (o ecotipos) de } \\
\text { cada especie } \\
\text { cultivada y silves- } \\
\text { tre presente en } \\
\text { cada subsistema. }\end{array}$} & $\begin{array}{l}\text { Número de subsistemas } \\
\text { presentes en el predio } \\
\text { (máximo 5). }\end{array}$ & $0-100$ \\
\hline & & & & $\begin{array}{l}\text { Número de especies } \\
\text { en relación con el máximo } \\
\text { de especies encontrado. }\end{array}$ & $0-100$ \\
\hline & & & & $\begin{array}{l}\text { Número de variedades en } \\
\text { relación con el máximo de } \\
\text { variedades encontrado en } \\
\text { los subsistemas. }\end{array}$ & $0-100$ \\
\hline
\end{tabular}


Tabla 1. Variables utilizadas en el análisis de riesgo socioecológico, técnicas de evaluación y valores asignados a cada variable (continuación)

\begin{tabular}{|c|c|c|c|c|c|}
\hline & Variable & Método y fuente & Descripción & Descripción del indicador & Valor \\
\hline \multirow{9}{*}{ 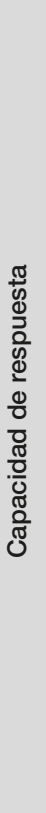 } & \multirow[t]{3}{*}{$\begin{array}{l}\text { Nivel de recu- } \\
\text { peración }\end{array}$} & \multirow{3}{*}{$\begin{array}{l}\text { Formulario sociopro- } \\
\text { ductivo, entrevista y } \\
\text { evaluación in situ. }\end{array}$} & \multirow{3}{*}{$\begin{array}{l}\text { Se considera si el } \\
\text { agricultor identifica } \\
\text { y utiliza especies } \\
\text { vegetales cultivadas } \\
\text { y/o variedades } \\
\text { (ecotipos locales) } \\
\text { que resistan o se } \\
\text { recuperen de perío- } \\
\text { dos de sequía. }\end{array}$} & $\begin{array}{l}\text { Identifica y usa especies } \\
\text { o variedades resistentes. }\end{array}$ & 100 \\
\hline & & & & $\begin{array}{l}\text { Identifica especies o varieda- } \\
\text { des, pero ya no las posee. }\end{array}$ & 50 \\
\hline & & & & $\begin{array}{l}\text { No identifica la presencia } \\
\text { de especies o variedades } \\
\text { resistentes. }\end{array}$ & 0 \\
\hline & \multirow{3}{*}{$\begin{array}{l}\text { Nivel de co- } \\
\text { nocimiento } \\
\text { y medidas } \\
\text { preventivas }\end{array}$} & \multirow{3}{*}{$\begin{array}{l}\text { Formulario sociopro- } \\
\text { ductivo, entrevista y } \\
\text { evaluación in situ. }\end{array}$} & \multirow{3}{*}{$\begin{array}{l}\text { Conocimiento y } \\
\text { medidas preventivas } \\
\text { para enfrentar esca- } \\
\text { sez de agua (técni- } \\
\text { cas de policultivo, } \\
\text { cosechas de agua, } \\
\text { almacenamiento de } \\
\text { grano, etc.). }\end{array}$} & $\begin{array}{l}\text { Realiza más de una técnica } \\
\text { o práctica. }\end{array}$ & 100 \\
\hline & & & & $\begin{array}{l}\text { Realiza al menos una técnica } \\
\text { o práctica. }\end{array}$ & 50 \\
\hline & & & & $\begin{array}{l}\text { No realiza técnicas o prác- } \\
\text { ticas. }\end{array}$ & 0 \\
\hline & \multirow[t]{3}{*}{$\begin{array}{l}\text { Redes de } \\
\text { apoyo }\end{array}$} & \multirow[t]{3}{*}{$\begin{array}{l}\text { Formulario sociopro- } \\
\text { ductivo y entrevista. }\end{array}$} & \multirow{3}{*}{$\begin{array}{l}\text { Si el agricultor cuenta } \\
\text { con redes sociales, } \\
\text { organizaciones } \\
\text { o instituciones (ONG, } \\
\text { gobierno u otras) que } \\
\text { lo apoyen en períodos } \\
\text { de escasez hídrica o } \\
\text { sequías. }\end{array}$} & $\begin{array}{l}\text { Alto (asistencia técnica } \\
\text { municipal, gobierno, ONG). }\end{array}$ & 100 \\
\hline & & & & $\begin{array}{l}\text { Medio (asistencia de una } \\
\text { institución o comunidad). }\end{array}$ & 50 \\
\hline & & & & Bajo (sin asistencia). & 0 \\
\hline
\end{tabular}

Fuente: elaboración propia.

\section{Cálculo del indice de riesgo socioecológico (IRSE)}

Para determinar valores de niveles de riesgo socioecológico a condiciones de escasez hídrica, fue utilizado el índice de riesgo socioecológico (IRSE), desarrollado con estos fines por Altieri (2013) y Montalba et al. (2013). Según lo señalado por Altieri (2013), esta metodología nos puede llevar a un modelo que nos permita establecer niveles de riesgo socioecológico en predios afectados por eventos climáticos extremos. Esta metodología considera la relación algorítmica entre amenaza, vulnerabilidad y capacidad de respuesta. El algoritmo de cálculo fue modificado respecto a los trabajos de Altieri (2013) y Montalba et al. (2013). Este índice modificado presenta una distribución normal y sus valores varían entre 0 y 2 , donde 0 indica bajos niveles de RSE, y ocurre lo contrario con el valor máximo 2. El IRSE se calculó utilizando la siguiente ecuación:

Donde:

Amenaza $=$ promedio (intensidad de la sequia + frecuencia + pérdida de rendimiento).

Vulnerabilidad $=$ promedio $($ porcentaje de plantaciones + acceso al agua + ubicación predial + contexto agroecológico). 
Capacidad de respuesta $=$ promedio (nivel de recuperación + nivel de conocimiento + redes de apoyo).

\section{Análisis estadistico}

Todas las variables analizadas se describieron mediante histogramas para facilitar su interpretación. Para el análisis estadístico, no se consideraron los predios de origen étnico correspondientes a colonos en Curarrehue debido a su bajo número. Para evaluar la amenaza, se promediaron los valores de todas las variables relacionadas con este componente para cada predio evaluado (ver la tabla 1). Lo mismo se realizó con las variables de vulnerabilidad y capacidad de respuesta. El efecto del origen étnico en estos tres componentes fue valorado utilizando la prueba post hoc de Tukey HSD (Tukey Honest Significant Difference). La presencia de autocorrelación espacial en los valores para cada variable analizada fue evaluada mediante el Mantel test con 999 iteraciones. Todos los análisis estadísticos se realizaron con el programa R v.2.15.0 (R Development Core Team, 2012).

\section{Resultados y discusión}

\section{Riesgo socioecológico}

Dados los valores obtenidos para cada una de las variables asociadas a la vulnerabilidad, a la amenaza y a la capacidad de respuesta, el cálculo del índice de riesgo socioecológico (IRSE) permitió evidenciar que la totalidad de los 177 casos estudiados presentan valores menores a 1,2 (estimado como un valor medio) y casi un 50\% presenta valores menores a 0,7 , lo cual puede ser considerado bajo nivel de riesgo socioecológico (figura 2).

Los valores de IRSE encontrados condicen con la realidad de la zona de estudio. La crisis del petróleo y las alzas del precio del dólar en la década de 1980, sumadas a la bajada del precio de los granos y los productos agrícolas, hicieron inviables gran parte de los sistemas cerealeros medianos y grandes de la zona, por cuyo motivo numerosos agricultores endeudados vieron como única alternativa posible el vender sus tierras a empresas forestales (generalmente, a bajo precio). Subvencionadas por el Estado de Chile por medio del Decreto 701 (1978), de fomento forestal, estas empresas establecieron extensas superficies de plantaciones para abastecer la industria de la celulosa, promovida por las políticas económicas instauradas en Chile por el gobierno militar de Pinochet. A diferencia de lo ocurrido en estas unidades de agricultura "empresarial moderna», una parte importante de los sistemas campesinos lograron sobrevivir a este proceso (Montalba, 2011; Montalba y Stephens, 2014), y se mantienen hasta hoy día en la zona de estudio. En consecuencia, los bajos niveles de riesgo socioecológico encontrados en las unidades campesinas estudiadas se relacionaría con su capacidad de amortiguar, resistir y generar estrategias individuales y/o colectivas para resistir perturbaciones sociales, económicas, políticas y ambientales, y sobrevivir a ellas, lo cual permitiría plantear que estas unidades campesinas tienen una mayor resiliencia y, más específicamente, resiliencia socioecológica (Hammond et al., 2013). 
Figura 2. Histograma de IRSE calculados para 177 unidades de agricultura tradicional campesina en la región de La Araucanía (Chile).

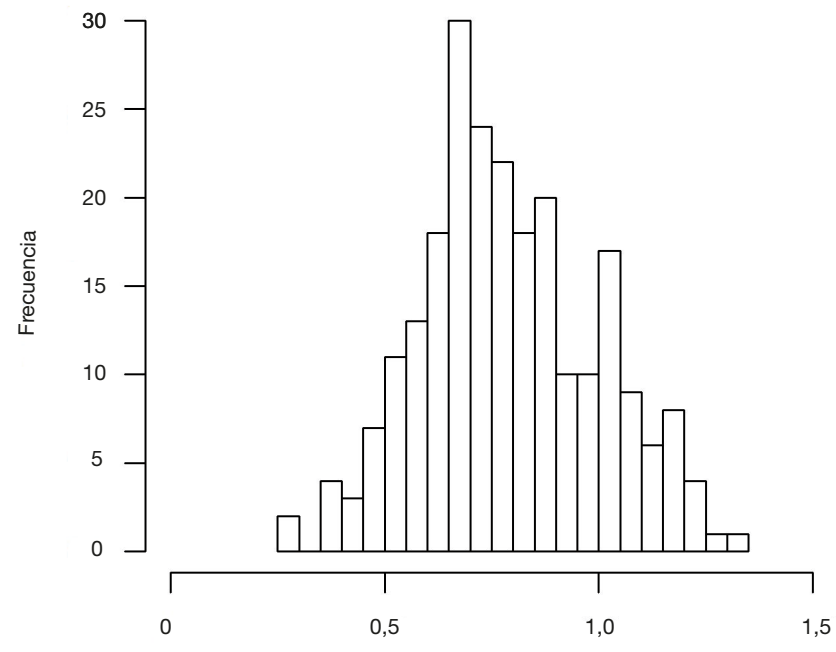

El origen étnico de los campesinos de las distintas unidades agrícolas estudiadas estaría relacionado con los niveles de amenaza, vulnerabilidad y capacidad de respuesta (figura 3). En consecuencia, es una variable que incide sobre el nivel de riesgo sociecológico y de resiliencia de estos sistemas. De esta forma, resulta destacable que las unidades prediales de Lumaco manejadas por agricultores de origen mapuche presentaran valores más altos de amenaza y capacidad de respuesta. Para la vulnerabilidad, estos mismos predios manejados por agricultores mapuches y de origen europeo presentaron valores más bajos que los predios manejados por agricultores chilenos. En el caso de Curarrehue, solo se encontró incidencia del origen étnico en la dimensión de capacidad de respuesta, ya que los predios manejados por agricultores mapuches presentaron valores más altos. En Lumaco, los altos valores en la dimensión amenaza que presentan los predios manejados por agricultores mapuches se explicarían por una mayor intensidad de la sequía y una mayor pérdida de rendimiento en caso de eventos de sequía para este grupo, en relación con las unidades manejadas por colonos europeos y chilenos (Tukey HSD $p<0,05)$. La frecuencia de sequías no presentó diferencias entre grupos. En cuanto a la menor vulnerabilidad de las unidades agrícolas manejadas por agricultores mapuches de Lumaco, esta se explicaría porque las unidades productivas manejadas por agricultores mapuches tienen una menor presencia de plantaciones forestales (Pinus radiata y Eucalyptus) en un radio de $1 \mathrm{~km}$ que las unidades manejadas por colonos europeos y chilenos (Tukey HSD $p<0,05$ ), aunque, en parte, esto se compensa por un valor menor en la variable de ubicación predial para unidades mapuches y chilenas en relación con colonos europeos (Tukey HSD $p<0,05$ ). Las otras variables 
Figura 3. Valores medios (+ error estándar de la media) de amenaza, vulnerabilidad y capacidad de respuesta para los predios evaluados en Lumaco y Curarrehue.

Amenaza
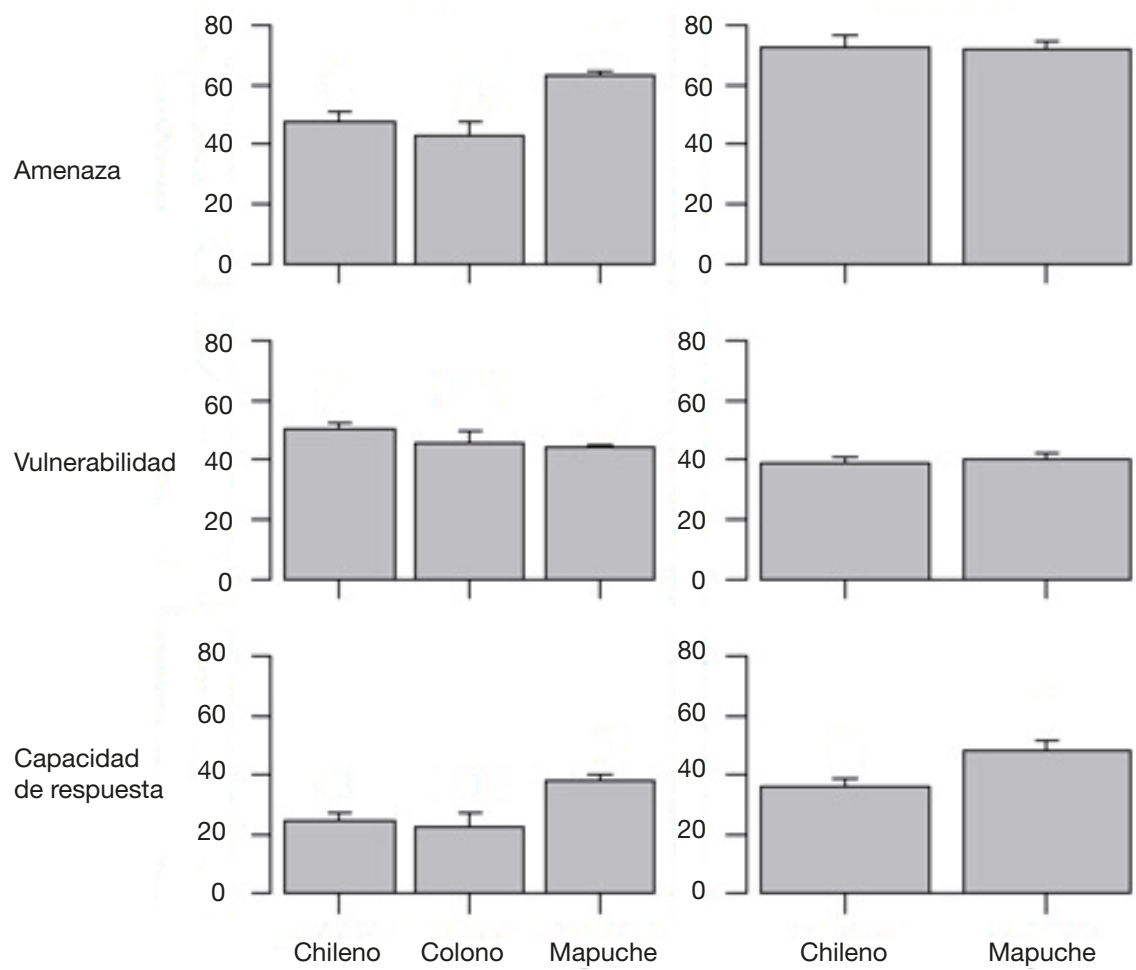

Las letras denotan diferencias significativas (Tukey $p<0,05$ ).

Fuente: elaboración propia.

de acceso al agua y contexto agreoecológico no presentaron diferencias según el grupo étnico. La mayor capacidad de respuesta de las unidades mapuches en Lumaco se explica por:

a) Un mayor nivel de recuperación de este grupo frente a los demás (Tukey $\mathrm{HSD} p<0,05)$.

b) Un mayor conocimiento de los mapuches frente a los colonos europeos. Sin embargo, las unidades manejadas por chilenos y colonos europeos presentan una mayor red de apoyo que las unidades manejadas por los agricultores mapuches (Tukey HSD $p<0,05$ ).

En Curarrehue la mayor capacidad de respuesta de las unidades manejadas por mapuches se debería a que estos presentan un mayor nivel de conocimiento y una mayor red de apoyo que en las unidades manejadas por campesinos chile- 
nos (Tukey HSD $p<0,05)$. Sin embargo, el nivel de recuperación es levemente superior en el caso de los chilenos (Tukey HSD $p=0,057$ ).

Estos resultados concuerdan con los de otros estudios realizados en procesos de modernización agrícola, en los cuales las diversidades biológica, productiva y cultural han desempeñado un rol importante en la reducción de riesgos socioecológicos, en el incremento de la resiliencia ante distintos factores de estrés y en la sustentabilidad. Como ejemplo de esto, Sendzimir et al. (2011) reportaron el fracaso de las políticas de modernización agrícola en el incremento de la resiliencia sociocultural en zonas de Níger y mostraron cómo los agricultores debieron recuperar prácticas culturales y sistemas diversificados de producción para aumentar su resiliencia. Del mismo modo, Baival (2012) reporta una situación similar ocurrida en sistemas de producción animal en Mongolia. De acuerdo con Maleksaeidi y Karami (2013), la resiliencia en sistemas socioecológicos (como los sistemas agrícolas) se vale de la utilización de aprendizaje social y de la innovación para incorporar diversos mecanismos de vida y aprendizaje de los cambios y la ocurrencia de situaciones perturbadoras e inesperadas. De esa forma, la reducción de riesgos socioecológicos y la mayor resiliencia sería, a su vez, una precondición para la sustentabilidad.

\section{Conclusiones}

A diferencia de lo planteado desde las fuentes oficiales, que consideran que los sistemas agrícolas tradicionales de características campesinas presentan altos niveles de riesgo socioecológico y bajos niveles de resiliencia a cambios globales ambientales, por medio de los resultados obtenidos en la presente investigación, planteamos que dichas consideraciones son parciales, dado que solo consideran en su análisis los rendimientos productivos de una especie (monocultivo), pero sin incluir variables más complejas de tipo socioecológico y culturales, las cuales evidenciarían un proceso histórico de cambio y adaptación a condiciones adversas, principalmente, en los sistemas agrícolas campesinos que han desarrollado prácticas culturales, estrategias agroecológicas y formas de organización socioproductiva, que conferirían mayor resiliencia socioecológica a sus sistemas productivos para enfrentar los cambios globales ambientales.

Posiblemente, debido a los distintos procesos históricos de asentamiento en el territorio y a los distintos modos culturales de vinculación entre los seres humanos y la naturaleza, la resiliencia socioecológica a condiciones de escasez hídrica de las unidades campesinas estudiadas presentaron valores diferenciables asociados al origen étnico de los grupos presentes en el territorio estudiado (mapuches, chilenos y colonos extranjeros). Del mismo modo, se considera que los niveles de agrobiodiversidad de cultivos alimenticios encontrados en los distintos grupos campesinos de La Araucanía, así como los sistemas de conocimiento y las estructuras socioproductivas vinculadas a su conservación, se encuentran directamente relacionados con su alta resiliencia socioecológica. 


\section{Agradecimientos}

A IDRC Canadá y a Fondecyt Chile, por su financiamiento por medio de los proyectos 106963-001 y 1141249, respectivamente; a los estudiantes de pregrado y postgrado, y a los agricultores que participaron en la etapa de investigación sobre el terreno.

\section{Referencias bibliográficas}

Altieri, M. (1999). Agroecología, bases cientificas para una agricultura sustentable. Montevideo: Editorial Nordan-Comunidad.

- (2013). «Construyendo resiliencia socio-ecológica en agroecosistemas: Algunas consideraciones conceptuales y metodológicas». En: Agroecología y resiliencia socioecológica: Adaptándose al cambio climático. Bogotá: Universidad Nacional de Colombia, 94-104.

Altieri, M. y Nicholls, C. (2009). «Cambio climático y agricultura campesina: Impactos y respuestas adaptativas». LEISA: Revista de Agroecología, 14, marzo, 5-8.

Altieri, M.; Nicholls, C. y Montalba, R. (2014). «El papel de la biodiversidad en la agricultura campesina en America Latina». LEISA, 30 (1), 5-9.

Altieri, M.; Nicholls, C. y Ríos, L. (2012). Agroecologia y resiliencia socioecologica: Adaptándose al cambio climático. Lima: Sociedad Científica de Agroecología (SOCLA).

Athanasiou, T. (1996). Slow Reckoning: The Ecology of a Divided Planet. Londres: Secker and Warburg.

Barval, B. (2012). Comunity-based Rangelan management and social-ecological resilience of rural mongolian communities. Colorado States University. Tesis doctoral.

Bauman, Z. (2006). Vida líquida. Barcelona: Paidós Ibérica.

Beck, U. (2006). La sociedad del riesgo: Hacia una nueva modernidad. Barcelona: Paidós Ibérica.

Brenner, C. (1997). Biotechnology Policy for Developing Country Agriculture [en línea]. París: OECD. $<$ http://dx.doi.org/10.1787/20771681>.

CASWELL, J.A. (2000). «An evaluation of risk analysis as applied to agricultural biotechnology (with a case study of GMO labeling)». Agribusiness [en línea], $16,115-123$. <http://dx.doi.org/10.1002/(SICI)1520-6297(200024)>.

Conaf-Conama (2012). Catastro de uso de suelo y vegetación periodo 1993-2007. Monitoreo y Actualización. Región de la Araucanía, Santiago de Chile, Gobierno de Chile, p. 29.

FolKE, C. (2006). «Resilience: The emergence of a perspective for social-ecological systems analyses». Global Environmental Change [en línea], 16, 253-267. $<$ http://dx.doi.org/10.1016/j.gloenvcha.2006.04.002>.

FriedmanN, H. (1998). «A sustainable world food economy». En: Keil, R.; Bell, D.V.J.; Penz, P. y Fawcett, L. (eds.). Political Ecology: Global and Local. Londres: Routledge \& Kegan Paul, 19-45.

Giddens, A.; Bauman, Z.; Luhmann, N.; Beck, U. y Beriain, E. (1996). Las consecuencias perversas de la modernidad. Barcelona: Anthropos.

Guzmán, G.; González de Molina, M. y Sevilla, E. (2000). Introducción a la agroecología como desarrollo rural sustentable. Madrid: Mundi-Prensa. 
Hammond, B.; Berardi, G. y Green, R. (2013). «Resilience in Agriculture: Smalland Medium-Sized Farms in Northwest Washington State». Agroecology and Sustainable Food Systems [en línea], 37, 316-339. <http://dx.doi.org/10.1080/10440046.2012.746251>.

Holling, C.S. (1973). «Resilience and Stability of Ecological Systems». Annual Review of Ecology and Systematics [en línea], 4, 1-23. <http://dx.doi.org/10.1146/annurev.es.04.110173.000245>.

Josling, T. y TANGERMANn, S. (1999). «Implementation of the WTO agreement on agriculture and developments for the next round of negotiations». Eur. R. Agric. Econ. [en línea], 26, 371-388. <http://dx.doi.org/10.1093/erae/26.3.371>.

Maleksaeidi, H. y Karami, E. (2013). «Social-ecological resilience and sustainable agriculture under water scarcity». Agroecology and Sustainable Foods Systems [en línea], 37, 262-290. <http://dx.doi.org/10.1080/10440046.2012.746767>.

Montalba, R. (2011). "Historia de la transformación del sistema hombre en el medioambiente en el secano interior de la IX Región de Chile: Una aproximación agroecológica». Revista CUHUSO, 8, 18-38.

Montalba, R.; Fonseca, F. y Thomet, M. (2015). Escasez de agua en La Araucanía. Elementos para la gobernanza en contextos multiculturales. Chile: CETSUR. 196 p.

Montalba, R.; García, M.; Altieri, M.; Fonseca, F. y Vieli, L. (2013). «Utilización del índice holístico de riesgo (IHR) como medida de resiliencia socioecológica a condiciones de escasez de recursos hídricos: Aplicación en comunidades campesinas e indígenas de La Araucanía, Chile». Agroecologia, 8 (1), 63-70.

Montalba, R. y Stephens, N. (2014). "Evaluating the "Ecological Mapuche" Idea: A historical sketch of the human ecology of Chile's Araucania Region». Human Ecology [en línea], 42 (4), 637-643. <http://dx.doi.org/10.1007/s10745-014-9678-0>.

Nicholls, C. y Altieri, M. (2013). Agroecología y cambio climático: Metodologías para evaluar resiliencias socioecológicas en comunidades rurales. Medellín: Sociedad Científica de Agroecología (SOCLA).

Paillotin, G. (1998). "The impact of biotechnologyonthe agro-food sector». En: Proceedings of the OECD, The Future of Food: Long Term Prospects for the Agro-Food Sector. París: OECD, 71-89.

RAMAKRISHNAN, P.S. (1999). "The impact of globalisation on agricultural systems of traditional societies». En: Dragun, A.K. y Tisdell, C. (eds.). Sustainable Agriculture and Environment. Cheltenham: Edward Elgar, 185-200.

Rouanet, J.L.; Romero, O. y Demanet, R. (1988). "Áreas agroecológicas de la IX Región: Descripción». IPA-Carillanca, 7 (1), 18-24.

Schnaiberg, A. y Gould, K. (1994). Environment And Society: The Enduring Conflict. New York, St Martin's Press.

Sendzimir, J.; Reij, C.P. y Maagnuszewsky, P. (2011). «Rebulling resilience in the Sahel: Regreening in the Maradi en Zinder regions of Niger». Ecology and Society [en línea], 16 (3), 1. <http://dx.doi.org/10.5751/ES-04198-160301>.

Sevilla Guzmán, E. y González de Molina, M. (1990). «Ecosociología: Elementos teóricos para el análisis de la coevolución social y ecológica». Revista Española de Investigaciones Sociológicas [en línea], 52, 7-45. <http://dx.doi.org/10.2307/40183497>. 
VArgas, A. (2007). «Cambio climático, agua y agricultura». IICA, 1 (2), 13-23.

Young, O. R.; Berkhout, F.; Gallopin, G. C.; Janssen, M. A.; Ostrom, E. y van DER LeEuw, S. (2006). "The globalization of socio-ecological systems: An agenda for scientific research». Global Environmental Change, 16 (3), 304-316. <http://dx.doi.org/10.1016/j.gloenvcha.2006.03.004>.

VVAA (1999). Catastro y evaluación de recursos vegetacionales nativos de Chile. Santiago de Chile: Universidad Austral de Chile, Pontificia Universidad Católica de Chile y Universidad Católica de Temuco. Proyecto Conaf-Conama-Birf. 89 p.

VVAA (2010a). Climate Change 2007: Impacts, Adaptation and Vulnerability. Cambridge University Press.

VVAA (2010b). El cambio climático en el sector silvoagropecuario de Chile. Santiago de Chile: Gobierno de Chile. Ministerio Nacional de Agricultura (MINAGRI).

VVAA (2012). The R Project for Statistical Computing [en línea]. Viena: R Foundation for Statistical Computing. <http://www.r-project.org>. 